\title{
Almost Surjective Epsilon-Isometry in The Reflexive Banach Spaces
}

\author{
Minanur Rohman \\ Department of Islamic Studies, Faculty of Tarbiyah, STAI Ma'Had Aly Al-Hikam, \\ Malang, Indonesia \\ Email: miminanira@gmail.com
}

\begin{abstract}
In this paper, we will discuss some applications of almost surjective $\varepsilon$-isometry mapping, one of them is in Lorentz space ( $L_{p, q}$-space). Furthermore, using some classical theorems of $w^{*}$-topology and concept of closed subspace $\alpha$-complemented, for every almost surjective $\varepsilon$-isometry mapping $f: X \rightarrow Y$, where $Y$ is a reflexive Banach space, then there exists a bounded linear operator $T: Y \rightarrow X$ with $\|T\| \leq \alpha$ such that
\end{abstract}

$$
\|T f(x)-x\| \leq 4 \varepsilon
$$

for every $x \in X$.

Keywords: almost surjectivity; $\varepsilon$-isometry; Lorentz space; reflexive space.

\section{INTRODUCTION}

Suppose $X$ and $Y$ be real Banach spaces. A mapping $U: X \rightarrow Y$ is called an isometry if

$$
\|U(x)-U(y)\|_{Y}=\|x-y\|_{X}, \text { for all } x, y \in X \text {. }
$$

Mazur-Ulam showed that if $U$ is a surjective isometry, then $U$ is affine. In other word, a surjective isometry mapping can be translated. This result lead to the following definition.

Definition 1.1. Let $X$ and $Y$ be real Banach spaces. A mapping $f: X \rightarrow Y$ is called an $\varepsilon$ isometry if there exists $\varepsilon \geq 0$ such that

$$
\left|\|f(x)-f(y)\|_{Y}-\|x-y\|_{X}\right| \leq \varepsilon, \text { for all } x, y \in X .
$$

If $\varepsilon=0$, then 0 -isometry is just an isometry. There are other names of $\varepsilon$-isometry, some of them are nearisometry [13], approximate isometry ([1], [7]), and perturbed metric preserved [3]. To simplify, the norm fuction is just written as $\|\cdot\|$ without mentioning the vector space.

Definition 1.1 above begs a question, "for every $\varepsilon$-isometry $f$, does always exist an isometry mapping $U$ and $\gamma>0$ such that

for all $x \in X$ ?".

$$
\|f(x)-U(x)\| \leq \gamma \varepsilon
$$

This problem was first investigated by Hyers-Ulam in 1945 [7] (therefore, $\varepsilon$-isometry problems are also called as Hyers-Ulam problems) and they found that for every surjective $\varepsilon$ isometry mapping $f$ in the Euclidean spaces, there always exists a surjective isometry mapping $U$ 
satisfying (1.1) with $\gamma=10$. Some years later, D. G Bourgin studied $\varepsilon$-isometry mapping in the Lebesgue space with $\gamma=12$.

In 1983, Gevirtz delivered $\gamma=5$ which was hold for any Banach space $X$ and $Y$ [6]. This result was sharpened by Omladić and Šemrl [10].

Theorem 1.2. Let $X$ and $Y$ be Banach spaces and $f: X \rightarrow Y$ is a surjective $\varepsilon$-isometry, there exists a linear surjective isometry mapping $U: X \rightarrow Y$ such that

$$
\|f(x)-U(x)\| \leq 2 \varepsilon, \text { for every } x \in X .
$$

There are many applications of $\varepsilon$-isometry mapping, such as Dai-Dong who use $\varepsilon$-isometry mapping to determine the smoothness and rotundity of Banach spaces. Šemrl and Väisälä proposed a definition of almost surjective mapping as follows [13].

Definition 1.3. Let $f: X \rightarrow Y$ is a mapping, $Y_{1}$ is a closed subspace of $Y$, and $\delta \geq 0$. A mapping $f$ is called almost surjective onto $Y$ if for every $y \in Y_{1}$, there exists $x \in X$ with $\|f(x)-y\| \leq \delta$ and for every $u \in X$, there exists $v \in Y_{1}$ with $\|f(u)-v\| \leq \delta$.

Using Definition 1.3, Šemrl and Väisälä weakened the surjective condition become almost surjective [13] (see also [14]).

Theorem 1.4. Let $E$ and $F$ be Hilbert spaces and $f: E \rightarrow F$ is an almost surjective $\varepsilon$-isometry with $f(0)=0$ that satisfies

$$
\sup _{\|y\|=1} \lim _{|t| \rightarrow \infty} \inf \left\|t y-\frac{f(E)}{t}\right\|<1
$$

then there exists a linear surjective isometry $U: E \rightarrow F$ such that

$$
\|f(x)-U(x)\| \leq 2 \varepsilon, \text { for every } x \in E .
$$

Šemrl and Väisälä showed that this result is true for $E$ and $F$ are Lebesgue spaces [13]. Vestfrid decrease the value 1 become $1 / 2$ in (1.2) and is valid for any Banach space [15].

On the other hand, Figiel proved that for any isometry mapping $U: X \rightarrow Y$ with $U(0)=0$, there exists an operator $\phi: \overline{\operatorname{span}} U(x) \rightarrow X$ with $\|\phi\|=1$ such that $\phi^{\circ} U=I$, the identity on $X$ [5].

Using Figiel's theorem, Cheng et. al. Give the following lemma [2] (see also Qian [11]).

Lemma 1.5. If $f: \mathbb{R} \rightarrow Y$ is a surjective $\varepsilon$-isometry with $f(0)=0$, then there exists $\phi \in Y^{*}$ with $\|\phi\|=$ 1 such that

$$
|\langle\phi, f(t)\rangle-t| \leq 3 \varepsilon, \text { for any } t \in \mathbb{R} .
$$
is true [12].

Using Vestfrid's theorem and Lemma 1.5, Minan et. al. showed that the following lemma

Theorem 1.6. Let $X$ and $Y$ be real Banach spaces, $f: X \rightarrow Y$ is an $\varepsilon$-isometry with $f(0)=0$. If the mapping $f$ satisfies almost surjective condition, i. e.

$$
\sup _{y \in S_{Y}} \lim _{|t| \rightarrow \infty} \inf \left\|t y-\frac{f(X)}{t}\right\|<\frac{1}{2}
$$

then for every $x^{*} \in X^{*}$, there exists a linear functional $\phi \in Y^{*}$ with $\|\phi\|=\left\|x^{*}\right\|=r$ such that

$$
\left|\langle\phi, f(x)\rangle-\left\langle x^{*}, x\right\rangle\right| \leq 4 \varepsilon r, \text { for every } x \in X .
$$




\section{RESULTS AND DISCUSSION}

We will discuss some applications of Theorem 1.6. Therefore, this paper will be organized as follows. Firstly, we will discuss two applications of Theorem 1.6, one of them is to determine the stability of almost surjective $\varepsilon$-isometry in $L_{p, q}$-spaces (also called as Lorentz spaces). Next, we will discuss the stability of almost surjective $\varepsilon$-isometry in reflexive Banach spaces.

The used notations and terminology are standard. $w^{*}$ (pronounced "weak star") topology of the dual of normed space $X$ is the smallest topology of $X^{*}$ such that for every $x \in X$, the linear functional $x^{*} \rightarrow x^{*} x$ on $X^{*}$ is continuous.

The author is greatly indebted to anonymous referee for a number of important suggestions that help the author to simplify the proof.

\section{Applications of Theorem 1.6}

Let $X$ and $Y$ be Banach spaces and $Y \in \mathfrak{B}(X, Y)$. For bounded set $C \subset X$, is defined $\|T\|_{C}=$ $\sup \{\|T x\|: x \in C\}$. If there is $c \in C$ such that $\|T c\|=\|T\|_{C}$, the $T$ attains its supremum over $C$. Operator $T$ is called as norm-attaining operator.

Theorem 2.1. Let $X$ and $Y$ be real Banach spaces, $f: X \rightarrow Y$ is an almost surjective $\varepsilon$-isometry with $f(0)=0$ satisfies

$$
\sup _{y \in S_{Y}} \liminf _{|t| \rightarrow \infty}\left\|t y-\frac{f(X)}{t}\right\|<\frac{1}{2}
$$

then

$$
\left\|\sum \lambda_{i} f\left(x_{i}\right)\right\|+4 \varepsilon \geq\left\|\sum \lambda_{i} x_{i}\right\|
$$

for every $x_{1}, \ldots, x_{n} \in X$ and every $\lambda_{1}, \ldots, \lambda_{n} \in \mathbb{R}$, where $\sum_{i=1}^{n} \lambda_{i}=1$.

Proof. Let $x_{1}, \ldots, x_{n} \in X$ and $X_{n}=\operatorname{span}\left(x_{1}, \ldots, x_{n}\right)$. Banach spaces $X$ and $Y$ that satisfy (2.1) are strictly convex [12]. In addition, $X^{*}$ and $Y^{*}$ are $w^{*}$-compact convex, so that for every $x^{*} \in S_{X_{n}}{ }^{*}$, there exists a linear functional $\varphi_{x^{*}} \in S_{Y^{*}}$ such that

$$
\left|\left\langle\varphi_{x^{*}}, f(x)\right\rangle-\left\langle x^{*}, x\right\rangle\right| \leq 4 \varepsilon \text {, for every } x \in X_{n} .
$$

Since $f$ is an almost surjective $\varepsilon$-isometry, then for every $y \in S_{Y}$, there exists $x_{0} \in X$ such that $f\left(x_{0}\right)=y$. Therefore,

$$
\begin{aligned}
\left\|y-\frac{f(X)}{t}\right\| & =\left\|f\left(x_{0}\right)-\frac{f(t x)}{t}\right\| \\
& \geq\left\|\varphi_{x^{*}}\right\|\left\|f\left(x_{0}\right)\right\|-\left\|\varphi_{x^{*}}\right\|\left\|\frac{f(t x)}{t}\right\| \mid-\left\|x^{*}\right\|\left\|x_{0}-x\right\|+\left\|x^{*}\right\|\left\|x_{0}-x\right\| \\
& \geq\left\|\varphi_{x^{*}}\right\|\left\|f\left(x_{0}\right)\right\|-\left\|\varphi_{x^{*}}\right\|\|f(x)\| \mid-\left\|x^{*}\right\|\left\|x_{0}-x\right\|+\left\|x^{*}\right\|\left\|x_{0}-x\right\| \\
& \geq \sup _{x^{*} \in S_{X_{n}}}\left|\left\langle x^{*}, x_{0}-x\right\rangle\right|-\left|\left\langle x^{*}, x_{0}-x\right\rangle\right|+\left|\left\langle\varphi_{x^{*}}, f\left(x_{0}\right)\right\rangle-\left\langle\varphi_{x^{*}}, f(x)\right\rangle\right| \\
& =\sup _{x^{*} \in S_{X_{n}}}\left|\left\langle x^{*}, x_{0}-x\right\rangle\right|-\left|\left\langle x^{*}, x_{0}-x\right\rangle\right|+\left|\left\langle\varphi_{x^{*}}, f\left(x_{0}\right)-f(x)\right\rangle\right| \\
& \geq \sup _{x^{*} \in S_{X_{n}}}\left|\left\langle x^{*}, x_{0}-x\right\rangle\right|-\left|\left\langle x^{*}, x_{0}-x\right\rangle-\left\langle\varphi_{x^{*}}, f\left(x_{0}\right)-f(x)\right\rangle\right|
\end{aligned}
$$


Recall that $X$ and $Y$ are strictly convex, so $f\left(x_{0}\right)-f(x)=\sum_{i=1}^{n}\left|\lambda_{i}\right| f\left(x_{i}\right)$ and $\quad x_{0}-x=$ $\sum_{i=1}^{n}\left|\lambda_{i}\right| x_{i}$. Since $x^{*} \in S_{X_{n}{ }^{*}}$, i. e. $\left\|x^{*}\right\|=1$, then $x^{*}$ is norm attaining functional. Hence, $\sup \left|\left\langle x^{*}, \sum_{i=1}^{n}\left|\lambda_{i}\right| x_{i}\right\rangle\right|=\left\|x^{*}\left(\sum_{i=1}^{n}\left|\lambda_{i}\right| x_{i}\right)\right\|=\left\|\sum_{i=1}^{n}\left|\lambda_{i}\right| x_{i}\right\|$. Therefore, $x^{*} \in S_{X_{n}}{ }^{*}$

$$
\begin{aligned}
\left\|\sum_{i=1}^{n}\left|\lambda_{i}\right| f\left(x_{i}\right)\right\| & \geq\left\|\sum_{i=1}^{n}\left|\lambda_{i}\right| x_{i}\right\|-\left|\left\langle x^{*}, \sum_{i=1}^{n}\left|\lambda_{i}\right| x_{i}\right\rangle-\left\langle\varphi_{x^{*}}, \sum_{i=1}^{n}\left|\lambda_{i}\right| f\left(x_{i}\right)\right\rangle\right| \\
& =\left\|\sum_{i=1}^{n}\left|\lambda_{i}\right| x_{i}\right\|-\sum_{i=1}^{n}\left|\lambda_{i}\right|\left|\left\langle x^{*}, \sum_{i=1}^{n} x_{i}\right\rangle-\left\langle\varphi_{x^{*}}, \sum_{i=1}^{n} f\left(x_{i}\right)\right\rangle\right| \\
& =\left\|\sum_{i=1}^{n}\left|\lambda_{i}\right| x_{i}\right\|-\left|\left\langle x^{*}, \sum_{i=1}^{n} x_{i}\right\rangle-\left\langle\varphi_{x^{*}}, \sum_{i=1}^{n} f\left(x_{i}\right)\right\rangle\right| \\
& \geq\left\|\sum_{i=1}^{n}\left|\lambda_{i}\right| x_{i}\right\|-4 \varepsilon
\end{aligned}
$$

or

$$
\left\|\sum_{i=1}^{n}\left|\lambda_{i}\right| f\left(x_{i}\right)\right\|+4 \varepsilon \geq\left\|\sum_{i=1}^{n}\left|\lambda_{i}\right| x_{i}\right\| .
$$

Next, we will discuss an application of Theorem 1.6 in $L_{p, q}$-spaces. Let $L(\Omega)$ is an algebra of equivalence classes of real valued measured function over $\left(\Omega, \sum, \mu\right)$. The distribution $\delta_{f}(s)$ for $f \in L(\Omega)$ is defined as

$$
\delta_{f}(s):=\mu(|f|>s)
$$

$L_{p, q}$-spaces are collections of all measured function over $\Omega$ such that

$$
\|f\|_{p, q}:=\left(\frac{q}{p} \int_{0}^{\infty} f^{*}(t)^{q} t^{\left(\frac{q}{p}\right)-1} d t\right)^{\frac{1}{q}}<\infty
$$

where $f^{*}(t):=\inf \left\{s>0: \delta_{f}(s)<t\right\}, t>0$. If $p=q$, then $L_{p, p}(\Omega)$ is just Lebesgue space $L_{p}(\Omega)$. The following theorem shows that there exists an isomorphism operator [8] in $L_{p, q}$-spaces.

Theorem 2.2. Suppose that $1 \leq p, q \leq \infty, p \neq q, p \neq 1, p \neq 2$. Then there exists no weakly sequence $\left\{f_{k}\right\}_{k=1}^{\infty}$ in $L_{p, q}(0,1)$ such that for some $C>0$ and for any subsequence $\left\{f_{k}^{\prime}\right\}_{k=1}^{\infty}$ of $\left\{f_{k}\right\}_{k=1}^{\infty}$, the estimate

$$
C^{-1} N^{1 / p} \leq\left\|\sum_{k=1}^{N} f_{k}^{\prime}\right\|_{p, q} \leq C N^{1 / p}
$$

holds.

Now, using Theorem 2.2, the author will show that the following theorem is true.

Theorem 2.3. Let $X=L_{p, q}$ and $Y$ be Banach space. If $f: X \rightarrow Y$ is an almost surjective -isometry with $f(0)=0$, then there exists an operator $S: Y \rightarrow X$ with $\|S\|=1$ such that

$$
\|S f(x)-x\| \leq 4 \varepsilon \text {, for every } x \in X .
$$

Proof. From Theorem 2.2, then $B_{X^{*}}$ is a $w^{*}$-closed convex hull of $w^{*}$-exposed point in $L_{p, q}$. Let $t \in(0,1)$ and $x^{*} \in S_{X^{*}}$. Theorem 1.6 implies that there exists $\phi_{t} \in S_{Y^{*}}$ such that

$$
\left|\left\langle\phi_{t}, f(x)\right\rangle-\left\langle x_{t}^{*}, x\right\rangle\right| \leq 4 \varepsilon, \text { for every } x \in X .
$$


Let $\mathrm{S}: \mathrm{Y} \rightarrow \mathrm{X}$ is defined by $\mathrm{S}(\mathrm{y})=\left\langle\phi_{t}, f(x)\right\rangle e_{t}=x_{0}$. Clearly $\|S\|=1$ and

$$
\begin{aligned}
\|S f(x)-x\|_{p, q} & =\left(\frac{q}{p} \int_{0}^{1}\left(x_{0}(t)^{q}-x(t)^{q}\right) t^{\left(\frac{q}{p}\right)-1} d t\right)^{\frac{1}{q}} \\
& \leq \sup _{t \in(0,1)}\left|\left\langle\phi_{t}, f(x)\right\rangle-\left\langle x_{t}^{*}, x\right\rangle\right| \leq 4 \varepsilon .
\end{aligned}
$$

\section{Almost Surjective $\varepsilon$-Isometry in The Reflexive Banach Spaces}

A normed space is reflexive if the natural mapping $\pi(x)$ from $X$ into $X^{* *}$ which is defined by $\pi(x): f \rightarrow f(\mathrm{x})$ where $f \in X^{*}$, is onto $X^{* *}$, or $X \approx X^{* *}$. Therefore, every reflexive normed space is Banach space. In addition, every closed subspace of reflexive Banach space is reflexive [4].

Proposition 3.1 (Megginson [9], Proposition 1.11.11). Let $X$ be reflexive normed space. Every functional $x^{*} \in X^{*}$ is norm-attaining functional.

The following definitions are classic (for more detail see [4]). Let X be Banach space and $M \subset X$. The polar set of $\mathrm{M}$ is $M^{\circ}:=\left\{x^{*} \in X^{*}:\left\langle x^{*}, x\right\rangle \leq 1\right.$, for all $\left.x \in M\right\}$. Conversely, ${ }^{\circ} M^{\circ}:=\left\{x \in X:\left\langle x^{*}, x\right\rangle \leq 1\right.$, for all $\left.x^{*} \in M^{\circ}\right\}$. The annihilator of $M$ is $M^{\perp}:=\left\{x^{*} \in X^{*}:\left\langle x^{*}, x\right\rangle=\right.$ 0 , for all $x \in M\}$, and preannihilator $M^{\perp}$ is ${ }^{\perp} M^{\perp}:=\left\{x \in X:\left\langle x^{*}, x\right\rangle=0\right.$ for all $\left.x^{*} \in M^{\perp}\right\}$.

For an almost surjective $\varepsilon$-isometry mapping $\mathrm{f}: \mathrm{X} \rightarrow \mathrm{Y}$ with $\mathrm{f}(0)=0$ and $\varepsilon>0, C(f)$ is a closed convex hull of $\mathrm{f}(\mathrm{X}), \mathrm{E}$ is an annihilator of subspace $\mathrm{F} \subset Y^{*}$ where $\mathrm{F}$ is a set of all bounded functionals over $C(f)$, and

$$
M_{\varepsilon}:=\left\{\phi \in Y^{*}: \phi \text { is bounded by } \beta \varepsilon \text { over } C(f), \text { for } \beta>0\right\} .
$$

Obviously, $C(f)$ is symmetric, then $M_{\varepsilon}$ is linear subspace of $Y^{*}$ with $M_{\varepsilon}=\cup_{n=1}^{\infty} n C(f)^{\circ}$. Therefore, $E=\bigcap\{\operatorname{ker} \phi: \phi \in M\}$. The following lemma is easy to be proved (see [2]).

Lemma 3.2. Let $Y$ be Banach space, the following statements are equicalence.

(1) $C(f) \subset E+F$ for a bounded $B \subset Y$;

(2) $M_{\varepsilon}$ is $w^{*}$-closed;

(3) $M_{\varepsilon}$ is closed.

For every almost surjective $\varepsilon$-isometry $f$, is defined a linear mapping $\ell: X^{*} \rightarrow 2^{Y^{*}}$ by

$$
\ell x^{*}:=\left\{\phi \in Y^{*}:\left|\langle\phi, f(x)\rangle-\left\langle x^{*}, x\right\rangle\right| \leq \beta \varepsilon, \text { for } \beta>0 \text { and all } x \in X\right\} .
$$

Since $\ell$ is a linear mapping, the definition of $M_{\varepsilon}$ implies

$$
\begin{aligned}
& \ell 0=\left\{\phi \in Y^{*}:|\langle\phi, f(x)\rangle-\langle 0, x\rangle| \leq \beta \varepsilon\right\} \\
& =\left\{\phi \in Y^{*}:|\langle\phi, f(x)\rangle| \leq \beta \varepsilon\right\} \\
& \quad=\left\{\phi \in Y^{*}: \phi \text { is bounded by } \beta \varepsilon \text { over } C(f)\right\}=M_{\varepsilon}
\end{aligned}
$$

and

$$
\ell x^{*}=\ell\left(x^{*}+0\right)=\ell x^{*}+\ell 0 \supset \phi+\ell 0=\phi+M_{\varepsilon} .
$$

Lemma 3.3. If $x^{*} \neq y^{*}$, then $\ell x^{*} \cap \ell y^{*}=\emptyset$.

Proof. Suppose that $\ell x^{*} \cap \ell y^{*}$ is nonempty, then there exists $\varphi \in \ell x^{*} \cap \ell y^{*}$. Assume and

$$
\varphi_{x^{*}}=\left\{\varphi \in Y^{*}:\left|\langle\varphi, f(x)\rangle-\left\langle x^{*}, x\right\rangle\right| \leq \beta \varepsilon \text {, for all } x \in X\right)
$$




$$
\varphi_{y^{*}}=\left\{\varphi \in Y^{*}:\left|\langle\varphi, f(x)\rangle-\left\langle y^{*}, x\right\rangle\right| \leq \beta \varepsilon, \text { for all } x \in X\right) .
$$

For $x^{*} \neq y^{*}$, there exists $\varphi \in \ell x^{*} \cap \ell y^{*}$ such that $\varphi_{x^{*}}=\varphi_{y^{*}}$. This is impossible because $\varphi$ is a linear functional and the proof is complete.

Let $U \in \mathfrak{B}(X, Y)$. Then $U^{*} \in \mathfrak{B}\left(Y^{*}, X^{*}\right)$ is called an adjoint operator such that $\langle U x, f\rangle=$ $\left\langle x, U^{*} f\right\rangle$ for all $x \in X$. Two results below are classic.

Proposition 3.4 (Fabian et. al. [4], Proposition 2.6). Let $M$ is a closed subspace of Banach space $X$. Then $(X / Y)^{*}$ is isometric with $M^{\perp}$ and $M^{*}$ is isometric with $X^{*} / M^{\perp}$.

Theorem 3.5 (Megginson [9], Proposition 3.1.11). Let $X$ and $Y$ be normed spaces. If $U \in \mathfrak{B}(X, Y)$, then $U^{*}$ is $w^{*}$-to- $w^{*}$ continuous. Conversely, if $Q$ is $w^{*}$-to- $w^{*}$ linear continuous operator from $Y^{*}$ to $X^{*}$, then there exists $U \in \mathfrak{B}(X, Y)$ such that $U^{*}=Q$.

Now, using Proposition 3.4 and Theorem 3.5, we will show that the following theorem is true.

Theorem 3.6. Let $X$ and $Y$ be Banach spaces, $f: X \rightarrow Y$ is an almost surjective $\varepsilon$-isometry with $f(0)=$ 0 , and $M=\bar{\ell} 0$. Then

(1) $Q: X^{*} \rightarrow Y^{*} / M$, which is defined by $Q x^{*}=\ell x^{*}+M$, is linear isometry.

(2) If $M$ is $w^{*}$-closed, then $Q$ is an adjoint operator of $U: E \rightarrow X$ with $\|U\|=1$.

Proof. (1) From Lemma 3.3 and definition of $\ell$, it is clear that $Q$ is linear. Lemma $3.2 \mathrm{implies} M=$ $\bar{\ell} 0=\bar{M}_{\mathcal{\varepsilon}}$. For every $x^{*} \in X^{*}$,

From definition of $\ell x^{*}$ and $M_{\varepsilon}$, we have

$$
\begin{aligned}
\left\|Q x^{*}\right\| & =\inf \left\{\|\phi-m\|: \phi \in \ell x^{*}, m \in M\right\} \\
& =\inf \left\{\|\phi-m\|: \phi \in \ell x^{*}, m \in M_{\varepsilon}\right\} .
\end{aligned}
$$

$$
\left\|Q x^{*}\right\|=\inf \left\{\|\phi\|: \phi \in \ell x^{*}\right\} .
$$

Theorem 1.6 gives

$$
\inf \left\{\|\phi\|: \phi \in \ell x^{*}\right\} \leq\left\|x^{*}\right\| .
$$

To show the conversely, from definition of $\ell$, there exists $\beta>0$ such that

$$
\left|\langle\phi, f(x)\rangle-\left\langle x^{*}, x\right\rangle\right| \leq \beta \varepsilon \text {, for all } x \in X .
$$

For any $\delta>0$, we can choose $x_{0} \in S_{X}$ such that

For all $n \in \mathbb{N}$, we get

$$
\left\langle x^{*}, x_{0}\right\rangle>\left\|x^{*}\right\|-\delta .
$$

As $n \rightarrow \infty$,

$$
\left|\left\langle\phi, \frac{f\left(n x_{0}\right)}{n}\right\rangle-\left\langle x^{*}, x_{0}\right\rangle\right| \leq \frac{\beta \varepsilon}{n}
$$

$$
\|\phi\| \geq \lim \sup _{n}\left|\left\langle\phi, \frac{f\left(n x_{0}\right)}{n}\right\rangle\right|=\left\langle x^{*}, x_{0}\right\rangle>\left\|x^{*}\right\|-\delta \text {. }
$$


Since $\delta$ is arbitrary, then

$$
\|\phi\| \geq\left\|x^{*}\right\| .
$$

From (3.1) and (3.2), then $\|\phi\|=\left\|x^{*}\right\|$ which shows that $Q$ is an isometry.

(2) Since $M$ is $w^{*}$-closed, then $M=\left\{{ }^{\perp} M\right\}^{\perp}=\left\{{ }^{\perp} M_{\varepsilon}\right\}^{\perp}=E^{\perp}$. Therefore, $Y^{*} / M=Y^{*} / E^{\perp}$. From definitions of $E$ and $M_{\varepsilon}$, Proposition 3.4 implies $Y^{*} / E^{\perp}=E^{*}$.

We will prove that $\phi$ is $w^{*}$-to- $w^{*}$ continuous over the unit ball $B_{X^{*}}$. Let $I$ is an index set and $\alpha \in I$. Suppose there exists net $\left(x^{*}\right) \subset B_{X^{*}}$ which is $w^{*}$-converging to $x^{*} \in X^{*}$. Using Theorem 1.6, there exists net $\left(\phi_{\alpha}\right) \subset Y^{*}$ with $\left\|\phi_{\alpha}\right\|=\left\|x_{\alpha}^{*}\right\|=r_{\alpha} \leq 1$ such that

$$
\left|\left\langle\phi_{\alpha}, f(x)\right\rangle-\left\langle x_{\alpha}^{*}, x\right\rangle\right| \leq 4 \varepsilon r_{\alpha}, \text { for all } x \in X
$$

Since $\left(\phi_{\alpha}\right)$ is $w^{*}$-compact, then there exists $w^{*}$-limit point $\in Y^{*}$ such that

$$
\left|\langle\phi, f(x)\rangle-\left\langle x^{*}, x\right\rangle\right| \leq 4 \varepsilon r, \text { for all } x \in X
$$

Therefore, $Q x_{\alpha}^{*}=\ell x_{\alpha}^{*}+M=\phi_{\alpha}+M$ is $w^{*}$-convergence to $\phi+M=Q x^{*}$. This result shows that $Q: B_{X^{*}} \rightarrow E^{*}$ is $w^{*}$-to- $w^{*}$ continuous. From Theorem 3.5, there exists $U: E \rightarrow X$ such that $U^{*}=Q$. Definition of $E$ shows that $U$ is a surjective mapping. Since $U^{*}=Q$ is a linear isometry, then $\|U\|=1$.

Before discussing the stability of an almost surjective $\varepsilon$-isometry in reflexive Banach space, we need some resuls below.

Proposition 3.7 (Fabian et. al. [4], Proposition 3.114). Let $Y$ be reflexive Banach space. If $M$ is a closed subspace of $Y$, then $M$ is Banach space.

Corollary 3.8. If $Y$ be reflexive Banach space, then the mapping $Q$ in Theorem 3.6 is an adjoint operator.

Proof. Since $Y$ is reflexive, from Proposition 3.7, $M$ is $w^{*}$-closed. The proof can be completed by Theorem 3.6.

Definition 3.9. Let $X$ be Banach space and $0 \leq \alpha<\infty$. A closed subspace $M \subset X$ is called $\alpha$ complemented in $X$ if there is a closed subspace $N \subset X$ with $M \cap N=\{0\}$ and projection $P: X \rightarrow$ $M$ along $N$ such that $X=M+N$ and $\|P\| \leq \alpha$.

Theorem 3.10. Let $X$ and $Y$ be Banach spaces where $Y$ is reflexive, and $f: X \rightarrow Y$ is an almost surjective $\varepsilon$-isometry, then there exists a bounded linear operator $T: Y \rightarrow X$ and $\|T\| \leq \alpha$ such that

$$
\|T f(x)-x\| \leq 4 \varepsilon, \text { for all } x \in X .
$$

Proof. Since $Y$ is reflexive, from Corollary 3.8 and Theorem 3.6, there exists a surjective operator $U: E \rightarrow X$ with $\|U\|=1$ such that $Q=U^{*}$. Since $E$ is $\alpha$-complemented in $Y$, there is a closed subspace $F \subset Y$ and $E \cap F=\{0\}$ such that $E+F=Y$ and projection $P: Y \rightarrow E$ along $F$ satisfies $\|P\| \leq \alpha$. Let $T=U P$, then

$$
\|T\|=\|U P\| \leq\|U\|\|P\|=\|P\| \leq \alpha .
$$

Furthermore,

$$
\left\langle Q x^{*}, P y\right\rangle=\left\langle Q x^{*}, y\right\rangle
$$

for all $x^{*} \in X^{*}$ and $y \in Y$. Therefore, 


$$
\left|\left\langle Q x^{*}, P f(X)\right\rangle-\left\langle x^{*}, x\right\rangle\right|=\left|\left\langle Q x^{*}, f(X)\right\rangle-\left\langle x^{*}, x\right\rangle\right| \leq 4 \varepsilon\left\|x^{*}\right\|,
$$

for all $x \in X$ and $x^{*} \in X^{*}$. Definition of adjoint operator gives

$$
\begin{aligned}
\left\langle Q x^{*}, \operatorname{Pf}(X)\right\rangle & =\left\langle x^{*}, Q^{*} \operatorname{Pf}(X)\right\rangle \\
& =\left\langle x^{*}, \operatorname{UPf}(X)\right\rangle=\left\langle x^{*}, T f(X)\right\rangle .
\end{aligned}
$$

Substitute (3.4) into (3.3), then

$$
\left|\left\langle x^{*}, T f(X)\right\rangle-\left\langle x^{*}, x\right\rangle\right|=\left|\left\langle x^{*}, T f(X)-x\right\rangle\right| \leq 4 \varepsilon\left\|x^{*}\right\|
$$

for all $x \in X$ and $x^{*} \in X^{*}$. In addition,

$$
\begin{aligned}
\left|\left\langle x^{*}, T f(X)-x\right\rangle\right| & \leq\left\|x^{*}\right\|\|T f(X)-x\| \\
& \leq 4 \varepsilon\left\|x^{*}\right\|
\end{aligned}
$$

or

$$
\|T f(X)-x\| \leq 4 \varepsilon
$$

for all $x \in X$.

\section{CONCLUSION}

In this paper, the author has shown simple applications of Theorem 1.6. In addition, almost surjective $\varepsilon$-isometries are remain stable in the reflexive Banach spaces.

Open Problem 1. This paper has shown that almost surjective $\varepsilon$-isometries are stable in Lorentz spaces when the conditions in Theorem 2.2 are satisfied. Is it possible to generalize these restrictions?

Open Problem 2. According to the results of this paper, is it possible to determine the stability of almost surjective $\varepsilon$-isometries in the higher dual of Banach spaces?

\section{REFERENCES}

[1] D. G. Bourgin, "Approximate isometries", Bull. Amer. Math. Soc., vol. 52, no. 8, pp. 704-713, 1954.

[2] L. Cheng, Y. Dong, and W. Zhang, "On stability of non-linear non-surjective $\varepsilon$-isometries of Banach spaces", J. Funct. Anal., vol. 264, no. 3, pp. 713-734, 2013.

[3] L. Cheng and Y. Zhou, "On perturbed metric-preserved mapping and their stability characteristic", J. Funct. Anal., vol. 264, no. 8, pp. 4995-5015, 2014.

[4] M. Fabian, P. Habala, P. Hájek, V. Montesinos, and V. Zizler, Banach Space Theory: The Basis for Linear and Nonlinear Analysis, Springer, New York, 2010.

[5] T. Figiel, "On nonlinear isometric embedding of normed linear spaces", Bull. Acad. Polon. Sci. Ser. Sci. Math. Astronom. Phys., vol. 16, pp. 185-188, 1968.

[6] J. Gevirtz, "Stability of isometries on Banach spaces", Proc. Amer. Math. Soc., vol. 89, no. 4, pp. 633-636, 1983.

[7] D. H. Hyers and S. M. Ulam, "On approximate isometries", Bull. Amer. Math. Soc., vol. 51, no. 4, pp. 288-292, 1945.

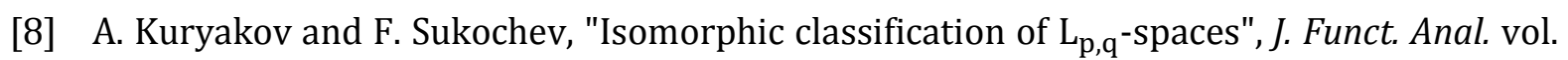
269, no. 8, pp. 2611-2630, 2015. 
[9] R. E. Megginson, An Introduction to Banach Space Theory, Graduate Text in Mathematics 183, Springer, New York, 1989.

[10] M. Omladič and P. Šemrl, "On nonlinear perturbation of isometries", Math. Ann. vol. 303, no. 4, pp. 617-628, 1995.

[11] S. Qian, "ع-isometries embedding", Proc. Amer. Math. Soc., vol. 123, no. 6, pp. 1797-1803, 1995.

[12] M. Rohman, R. B. E. Wibowo, and Marjono, "Stability of an almost surjective epsilonisometry in the dual of real Banach spaces", Aust. J. Math. Anal. Appl., vol. 13, no. 1, pp. 1-9, 2016.

[13] P. Šemrl and J. Väisälä, "Nonsurjective nearisometries of Banach spaces", J. Funct. Anal., vol. 198, no. 1, pp. 268-278, 2003.

[14] I. A. Vestfid, "Almost surjective $\varepsilon$-isometries of Banach spaces", Colloq. Math., vol. 100, no. 1, pp. 17-22, 2004.

[15] I. A. Vestfrid, "Stability of almost surjective $\varepsilon$-isometries of Banach spaces", J. Funct. Anal., vol. 269, no. 7, pp. 2165-2170, 2015. 